\title{
Remarkably high miscibility of octa-substituted POSS with commodity conjugated polymers and molecular fillers for the improvement of homogeneities of polymer matrices
}

\author{
Kazunari Ueda ${ }^{1,2}$, Kazuo Tanaka ${ }^{1}$ and Yoshiki Chujo ${ }^{1}$
}

This manuscript describes the extremely high affinities of polyhedral oligomeric silsesquioxane (POSS) with conjugated polymers. Polymer films of polyfluorene (PF) and poly(3-hexylthiophene) (P3HT) containing a series of octa-substituted POSS at variable concentrations were prepared. The relationships between the thermal and optical properties of commodity-conjugated polymers and the chemical structures of the substituents in POSS were evaluated by a series of experiments. It was shown that the POSS fillers with relatively longer alkyl substituents can enhance the thermal stabilities of the polymer matrices, even though most of the POSS fillers reduced the decomposition temperatures. The UV - Vis absorption measurements showed that the narrower absorption bands were obtained from the POSS-containing film with PF. It was suggested that the aggregation of the polymer chains could be suppressed by the POSS filler, leading to highly homogeneous states in the films. Moreover, for the P3HT films, the extension of the main-chain conjugation was induced by the POSS fillers with shorter alkyl substituents via the suppression of intermolecular interactions among the polymer chains. These data mean that the POSS fillers can have a significant role in the improvement of the homogeneity in the polymer films due to their high affinity to the conjugated systems.

Polymer Journal (2016) 48, 1133-1139; doi:10.1038/pj.2016.84; published online 28 September 2016

\section{INTRODUCTION}

The use of the printing method reduces the production cost of large-sized flat displays and enables the fabrication of paper-like opto-electronic devices. Conjugated polymers are regarded as a feasible candidate for use as the 'ink' in the printing method because of their optical properties and carrier-transport abilities. ${ }^{1}$ Thus, the development of functional conjugated polymers with optical and electronic functionalities is a topic of high relevance. ${ }^{2,3}$ However, the intrinsic lower stabilities of the polymeric materials compared with those of inorganics are still a critical limitation to the practical application of functional conjugated polymers. Thus, high chemical and thermal stabilities as well as optical and electrical properties are required for the application of these polymers in modern organic devices.

The formation of organic-inorganic polymer hybrids is a facile strategy for enhancing the thermal and mechanical properties of polymers and for obtaining functional materials with high stability. ${ }^{4,5}$ When the domain size of the polymer components in the mixture can be maintained at the nanoscale, the hybrid-like homogeneous clear solids can be produced. ${ }^{6-9}$ The advantages of both the polymers (processability, flexibility, and function-tunability) and the inorganic materials (robustness and stability) are obtained simultaneously by the hybrid materials. The superior characteristics of the hybrids relative to those of the original materials have enabled the fabrication of advanced materials; for example, a series of optically functional hybrids have been manufactured such as white-light emissive materials, ${ }^{10}$ environmental-resistant conductors $^{11}$ and oxygen-resistant phosphorescent emitters. ${ }^{12}$ In general, the hybrid materials were prepared by a sol-gel method in the polar solvents. However, intrinsic low compatibility of polymers, especially conjugated polymers, not only with inorganic elements but also with the polar solvents, used in a conventional sol-gel method, is a critical issue for suppressing the aggregation of the polymer element in the materials and realizing the hybridization. Instead of a sol-gel method, we have recently proposed the use of the molecular filler composed of polyhedral oligomeric silsesquioxane (POSS) for the formation of hybrid materials. ${ }^{13,14}$ POSS has a symmetric structure with a rigid silica cube and organic substituents at the vertices, and various types of unique materials have been developed with POSS derivatives. ${ }^{15-30}$ Therefore, POSS derivatives have attracted attention as a versatile 'element-block', which is a functional building block composed of heteroatoms, for constructing advanced nanomaterials. ${ }^{31,32}$ Especially,

${ }^{1}$ Department of Polymer Chemistry, Graduate School of Engineering, Kyoto University, Kyoto, Japan and ${ }^{2}$ Matsumoto Yushi-Seiyaku Co., Ltd., Osaka, Japan Correspondence: Dr K Tanaka or Professor Y Chujo, Department of Polymer Chemistry, Graduate School of Engineering Kyoto University, Katsura, Nishikyo-ku 615-8510, Kyoto, Japan.

E-mail: kazuo123@chujo.synchem.kyoto-u.ac.jp or chujo@chujo.synchem.kyoto-u.ac.jp

Received 30 June 2016; revised 26 July 2016; accepted 1 August 2016; published online 28 September 2016 
if homogeneous dispersion can be realized with POSS in the polymer matrix, these mixtures will be identical to the conventional polymer hybrids. Indeed, the homogeneous polymer films with various types of POSS can be readily prepared by casting and spin-coating using the mixture solutions containing POSS and polymers in organic solvents. The resulting 'hybrids' showed higher thermal and mechanical properties than the pristine polymer samples. ${ }^{33,34}$ In particular, by modulating the chemical structures of the POSS substituents, multi-functions including high thermal stability can be obtained according to the preprogrammed design. ${ }^{35-40}$ For this research effort, the next challenge is to demonstrate the feasibility of the use of POSS fillers for enhancing the robustness of conjugated polymers without the loss of electronic properties.

Herein, high affinities of POSS with the commodity-conjugated polymers are reported. A series of octa-substituted POSS fillers with various types of alkyl groups were prepared and introduced into polymer films composed of polyfluorene (PF) and poly(3-hexylthiophene) (P3HT) commodity-conjugated polymers. The changes in the optical and thermal properties were monitored while using these hybrid films. First, the tremendously high dispersion abilities of POSS in the polymer matrices are demonstrated. Homogeneous films with several tens of weight-percent of POSS can be prepared. The thermal reinforcements by the longer alkyl-substituted POSS fillers were demonstrated. Next, the optical properties of these films were compared with those of the pristine polymer films. Accordingly, the suppression of the aggregation states in $\mathrm{PF}$ was suggested. Additionally, the concentration quenching of $\mathrm{P} 3 \mathrm{HT}$ was demonstrated. These data mean that the homogeneity in the conjugated polymer films could be improved by the POSS fillers. Structure-property relationships between the properties of the conjugated polymer films and the chemical structures of the POSS fillers are discussed.

\section{RESULTS AND DISCUSSION}

The chemical structures of the octa-substituted POSS used in this study as a filler are listed in Figure 1. The synthesis of a series of POSS fillers was performed according to the procedures described in previous studies. ${ }^{41} \mathrm{PF}$ and $\mathrm{P} 3 \mathrm{HT}$ were prepared via the Yamamoto and oxidation coupling reaction according to the previous methods, respectively (Supplementary Table S1). ${ }^{42-44}$ The polymer films containing the POSS fillers were prepared using solution blending of POSS fillers and each polymer. The mixture of the polymer in chloroform was added to the POSS fillers with various concentrations (up to $80 \mathrm{wt} \%$ ) and stirred at ambient temperature. The resulting mixtures were cast onto the quartz plate, and the film samples for the measurements were obtained after drying in an oven.

The surfaces of the cast films containing $40 \mathrm{wt} \%$ the POSS fillers were observed with a scanning electron microscopy (SEM; Supplementary Figure S1 in the Supporting Information). Uniform images were obtained from the observation. This result means that the significant phase separation or aggregation of POSS fillers hardly occurred in the films. Indeed, visual examination of the image does not show any critical inhomogeneities (Supplementary Figure S2). Moreover, based on the absorption spectra, it is clear that the

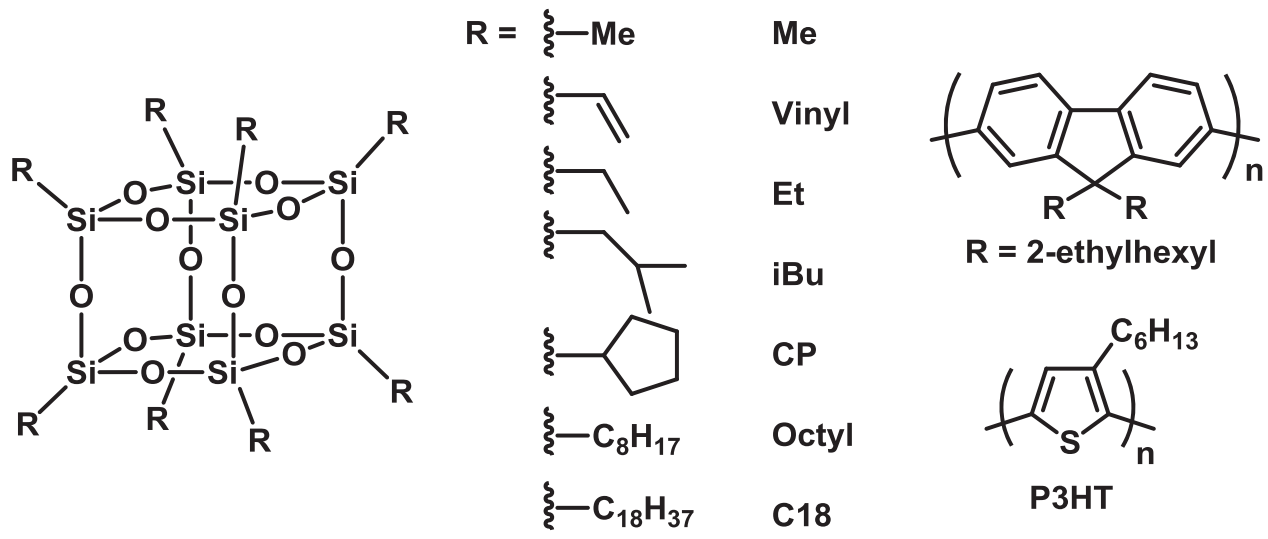

Figure 1 Chemical structures of the POSS fillers and conjugated polymers used in this study.

Table 1 Temperature changes $\left({ }^{\circ} \mathrm{C}\right)$ in $T_{\mathrm{d} 20^{\mathrm{S}}} \mathrm{S}$ of the polymer matrices obtained by adding various amounts of POSS fillers

\begin{tabular}{|c|c|c|c|c|c|c|c|c|c|}
\hline & \multicolumn{4}{|c|}{$P F^{a}$} & \multicolumn{4}{|c|}{ P3HT } & \multirow{2}{*}{$\frac{P O S S}{T_{d 20}}$} \\
\hline & $10 w t \%$ & $20 w t \%$ & $30 w t \%$ & $40 w t \%$ & $10 w t \%$ & $20 w t \%$ & $30 w t \%$ & $40 w t \%$ & \\
\hline Vinyl & -22 & -165 & -170 & -186 & -99 & -129 & -124 & -128 & 235 \\
\hline Et & -17 & -137 & -168 & -157 & -144 & -154 & -188 & -192 & 214 \\
\hline ¡Bu & -214 & -116 & -166 & -180 & -74 & -155 & -171 & -166 & 249 \\
\hline $\mathrm{C} 18$ & 2 & -3 & 2 & 6 & -68 & -29 & 40 & 58 & 465 \\
\hline
\end{tabular}

Abbreviations: P3HT, poly(3-hexylthiophene); PF, polyfluorene.

${ }^{\mathrm{a}} T_{\mathrm{d} 20}$ of the pristine film was $418^{\circ} \mathrm{C}$.

${ }^{\mathrm{b}} T_{\mathrm{d} 20}$ of the pristine film was $402{ }^{\circ} \mathrm{C}$ 

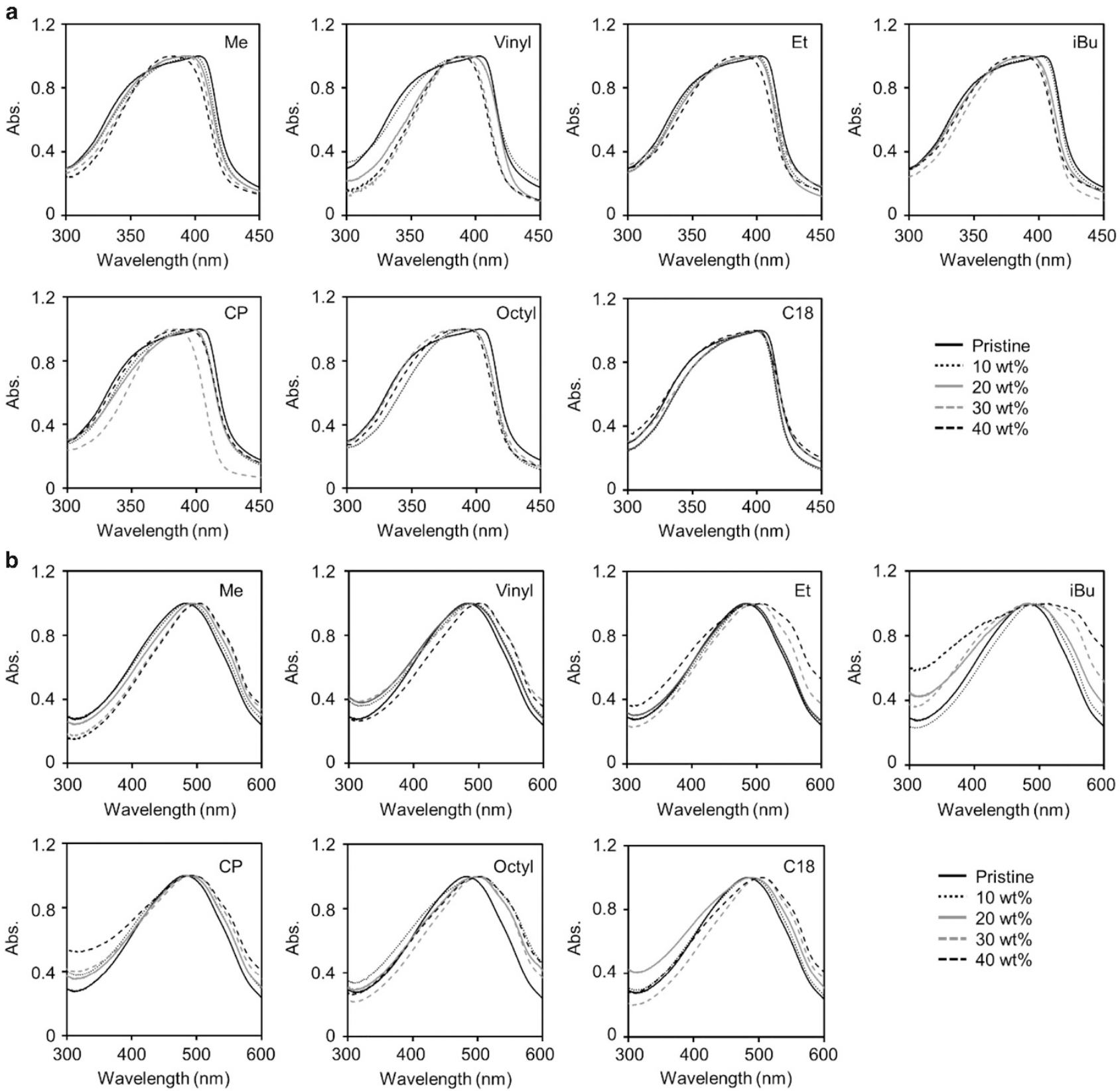

Figure 2 Absorption spectra of (a) the PF and (b) P3HT films containing variable amounts of POSS fillers.

Table 2 Peak shifts $(\Delta \lambda, \mathrm{nm})$ of absorption bands obtained by adding various amounts of POSS fillers

\begin{tabular}{|c|c|c|c|c|c|c|c|c|}
\hline & \multicolumn{4}{|c|}{$P F^{a}$} & \multicolumn{4}{|c|}{ PЗHT } \\
\hline & $10 w t \%$ & $20 w t \%$ & $30 w t \%$ & $40 w t \%$ & $10 w t \%$ & $20 w t \%$ & $30 w t \%$ & $40 w t \%$ \\
\hline Vinyl & -8 & -8 & -14 & -14 & 3 & 4 & 16 & 23 \\
\hline Et & -4 & -7 & -8 & -14 & 2 & 3 & 18 & 20 \\
\hline iBu & -2 & -10 & -12 & -16 & 7 & 12 & 18 & 25 \\
\hline C18 & -2 & -5 & -5 & -5 & 1 & 6 & 19 & 21 \\
\hline
\end{tabular}

Abbreviations: P3HT, poly(3-hexylthiophene); PF, polyfluorene. 
background was kept at a low level (Supplementary Figure S3). This implies that light dispersion should hardly occur in the films. These data suggest that sufficient transparency for measuring the optical properties with good reproducibility was maintained with $40 \mathrm{wt} \%$ POSS fillers. It should be mentioned that the films prepared by casting can maintain the transparency even with $40 \mathrm{wt} \%$ POSS fillers. In the case of commodity polymers such as polystyrene and poly(methyl methacrylate), turbidity appeared only upon addition of wt $\%$ of the POSS fillers. ${ }^{45}$ This means that POSS has good intrinsic compatibility with conjugated polymers. It was reported that the closed silica cage is strongly hydrophobic. ${ }^{46-49}$ It is likely that the cubic structure can interact strongly with the planar structures of the polymer main-chain. However, several samples showed turbidity at $60 \mathrm{wt} \%$, and the homogeneities of the films critically decreased. The phase separation observed in most of the films containing $80 \mathrm{wt} \%$ of the POSS fillers decreased the film-formability and significantly reduced the reproducibility of optical measurements. Thus, we discuss the filler effects with the data sets obtained from the samples containing $40 \mathrm{wt} \%$ POSS.

The thermal stabilities of the polymer films were evaluated using TGA (Table 1). Since significant thermal degradation was observed in the samples around the temperature with $20 \%$ weight losses $\left(T_{\mathrm{d} 20}\right)$ assigned as the main chain scissions, the thermal stabilizing effects of the POSS fillers were evaluated using the comparison of the $T_{\mathrm{d} 20}$ values. ${ }^{50}$ It was shown that the use of most POSS fillers induced the decreases of the $T_{\mathrm{d} 20}$ values of both polymer matrices. It is likely that the conjugated polymers usually form tight intermolecular interactions
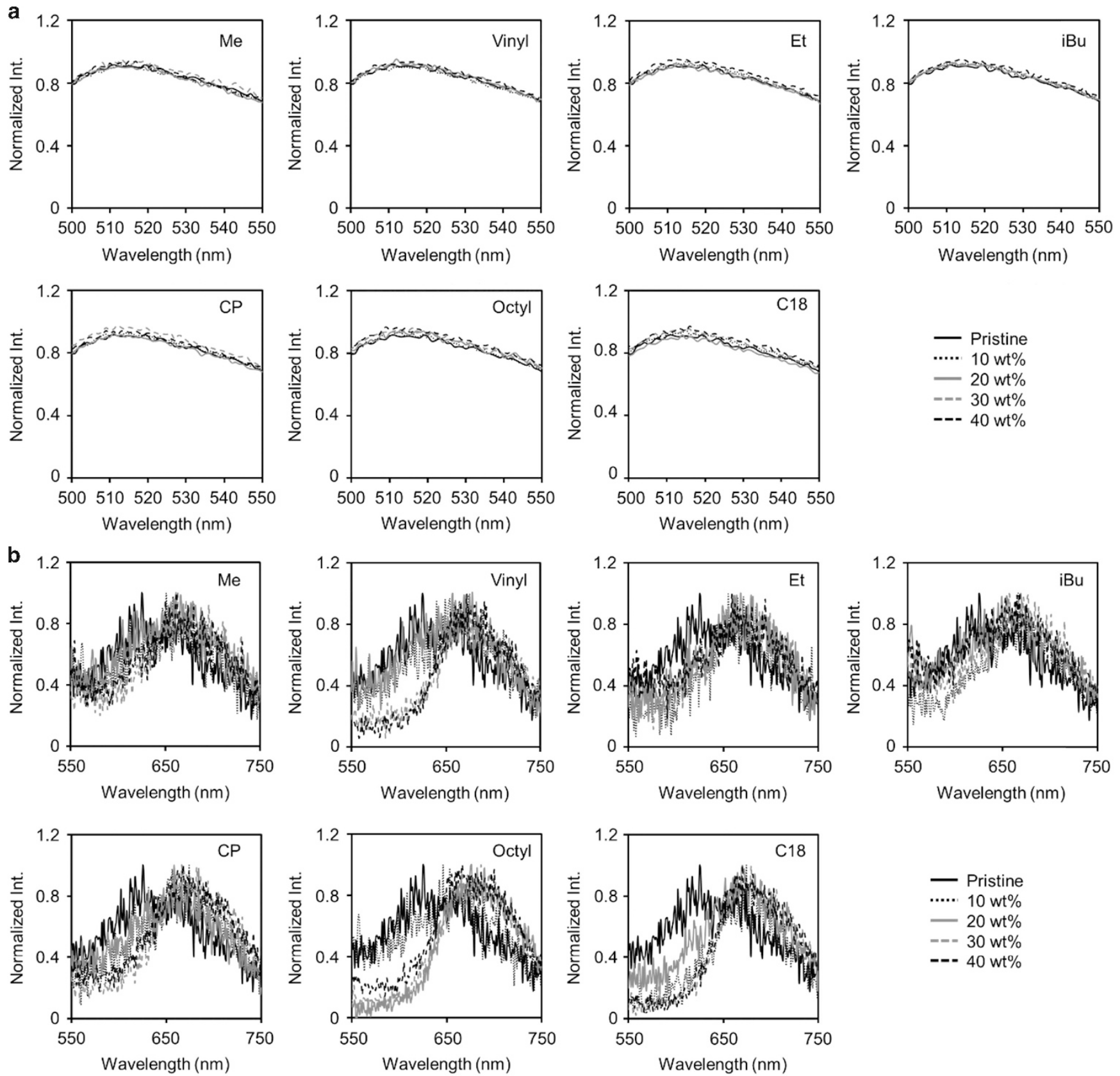

Figure 3 Emission spectra of (a) the PF and (b) P3HT films containing variable amounts of POSS fillers. 
such as the $\pi-\pi$ stacking. Cubic silica could be a suitable structure as a plasticizer. Therefore, a reduction of thermal stabilities was induced. In contrast, several POSS fillers can enhance the thermal stabilities of the polymer matrices. The $T_{\mathrm{d} 20}$ values of $\mathrm{PF}$ and $\mathrm{P} 3 \mathrm{HT}$ were elevated by $+6{ }^{\circ} \mathrm{C}$ and $+58{ }^{\circ} \mathrm{C}$ with $40 \mathrm{wt} \% \mathrm{C} 18$-POSS, respectively. The longer alkyl-chains can make a stronger interaction with the polymers. Then, the thermal vibration can be efficiently suppressed by POSS fillers. As a result, destabilization effects by the POSS core could be compensated. We also performed DSC measurements on the polymer films (Supplementary Table S2). In particular, decreases of the glass transition temperatures $\left(T_{\mathrm{g}}\right)$ were observed. These data implied that the POSS fillers could form highly dispersion states in the polymer matrices. Upon loading the POSS fillers with $20 \mathrm{wt} \%$ concentration, drastic decreases in $T_{\mathrm{d} 20}$ s were observed as shown in Table 1. According to the previous studies with poly(methyl methacrylate) and polystyrene, the maximum amounts of POSS fillers were $\sim 20 \mathrm{wt} \%{ }^{41,45}$ At this concentration level, critical phase separation was often observed in the composite samples, resulting in losses of film-formability and thermal stability. For the conjugated polymers, this implied that inhomogeneity could be induced at $20 \mathrm{wt} \%$ filler concentration, leading to irregular decreases in $T_{\mathrm{d} 20} \mathrm{~s}$ at $20 \mathrm{wt} \%$ filler concentrations even though significant changes in the film formation were hardly observed. In contrast, it should be mentioned that additional amounts of POSS fillers can be loaded in the conjugated polymers. In this case, larger improvements of thermal stabilities can be observed.

The optical properties of the polymer films were also investigated. Initially, the UV - Vis absorption spectra were measured for both the polymer films (Figure 2, Table 2). Generally, due to the intermolecular interactions in the condensed state, redshifts and peak broadening were often observed in the absorption spectra of the films compared with those of the solution. In particular, it is known that these interactions can be readily found in the condensed state of PF, leading to the formation of the $\beta$-phase that provides the characteristic absorption band in the longer wavelength region..$^{51,52} \mathrm{~A}$ broader absorption band was obtained for the cast film of PF, than for the film obtained by spin-coating. This result suggests that the $\beta$-phase should be typically produced in the pristine PF cast film. Interestingly, upon addition of the POSS fillers, the peak broadening was reduced, and blue shifts of the absorption band were observed. The introduction of the POSS fillers with relatively shorter alkyl and compact substituents (Me- and CP-POSS) induced obvious blue shifts in the spectra. The degree of the peak shift was then increased by increasing the amounts of the POSS fillers. It is proposed that the POSS fillers may be capable of disturbing the intermolecular interactions of polymer main-chains because the cubic structure of the POSS core could be favorable for the interaction with the conjugated polymer chains. Consequently, the aggregation could be released in the films. These data mean that homogeneity should be enhanced by the POSS fillers. The redshifts from the $\mathrm{P} 3 \mathrm{HT}$ films are discussed using the data including the emission properties.

To examine the influence of the POSS fillers on the luminescent properties of the polymer films, emission spectra were measured (Figure 3 and Tables 3 and 4). Significant effects were observed for the P3HT films. It was found that the redshifts of the emission bands were induced by increasing the POSS filler content. It was revealed that a drastic redshift of the emission band was observed for all films with $40 \mathrm{wt} \%$ POSS fillers. Relatively larger shifts were obtained from the longer alkyl-substituted POSS. It is known that P3HT can form a strong stacking in the film state because of the high planarity of the polymer chains. ${ }^{53-55}$ At this stage, each polymer chain should be structurally restricted. On the other hand, loading the POSS filler should isolate the polymer, leading to the release from these structural restrictions not only in the excited state but also in the ground state. Then, it is proposed that the polymer chains could form the energetically favorable forms for developing main-chain conjugation, leading to the drastically redshifted absorption and emission bands. Slight improvements in the emission efficiencies of the P3HT films were induced by the POSS fillers. In general, the emission efficiencies decreased in the film state via concentration quenching. This implied that the POSS filler may disturb the intermolecular interactions of the polymer main-chains, resulting in the release from the concentration quenching. It should be mentioned that C18-POSS induced the largest degree of peak shifts despite the improved thermal stability. This fact means that C18-POSS can improve the homogeneity of the film without the loss of thermal stability.

\section{CONCLUSION}

This work demonstrates the feasibility of the use of POSS fillers for modulating the properties of conjugated polymers, such as PF and P3HT, that are widely used as key materials in the modern organic opto-electronic devices. It was shown that a series of POSS can be mixed via the solution method in the polymer films with several tens weight percentages. Using thermal analyses, it was observed that the degradation temperatures of the polymer films were elevated by adding the POSS fillers with relatively longer alkyl chains. The optical measurements suggested that the POSS fillers with shorter alkyl chains showed a particular suppression of the formation of the polymer

Table 3 Peak shifts $(\mathrm{nm})$ of the emission bands obtained by adding various amounts of POSS fillers

$P F$

\begin{tabular}{|c|c|c|c|c|c|c|c|c|}
\hline & $10 w t \%$ & $20 w t \%$ & $30 w t \%$ & $40 w t \%$ & $10 w t \%$ & $20 w t \%$ & $30 w t \%$ & $40 w t \%$ \\
\hline $\mathrm{Me}$ & 1 & 4 & 2 & 5 & 32 & 35 & 35 & 36 \\
\hline Vinyl & 0 & 1 & 4 & 5 & 48 & 45 & 36 & 33 \\
\hline Et & 1 & 2 & 5 & 4 & 29 & 35 & 36 & 33 \\
\hline iBu & 0 & 4 & 2 & 3 & 40 & 32 & 40 & 33 \\
\hline $\mathrm{CP}$ & 3 & 0 & 1 & 3 & 26 & 32 & 37 & 36 \\
\hline Octyl & 1 & 2 & 4 & 3 & 31 & 48 & 39 & 40 \\
\hline C18 & 4 & 3 & 4 & 2 & 44 & 44 & 44 & 44 \\
\hline
\end{tabular}

Abbreviations: P3HT, poly(3-hexylthiophene); PF, polyfluorene.

aDetermined with the peak at $511 \mathrm{~nm}$

Determined with the peak at $626 \mathrm{~nm}$. 
Table 4 Emission efficiency changes (\%) obtained by adding various amounts of POSS fillers

\begin{tabular}{|c|c|c|c|c|c|c|c|c|}
\hline & \multicolumn{4}{|c|}{$P F^{a}$} & \multicolumn{4}{|c|}{ P3HT } \\
\hline & $10 w t \%$ & $20 w t \%$ & $30 w t \%$ & $40 w t \%$ & $10 w t \%$ & $20 w t \%$ & $30 w t \%$ & $40 w t \%$ \\
\hline $\mathrm{Me}$ & 5 & 3 & 0 & -1 & 0.3 & 0.5 & 0.6 & 0.7 \\
\hline Vinyl & 1 & 0 & 0 & 0 & 0.2 & 0.3 & 0.6 & 0.9 \\
\hline Et & 2 & 3 & 1 & 3 & 0.1 & 0.2 & 0.6 & 0.7 \\
\hline iBu & 3 & 1 & 3 & 0 & 0.4 & 0.5 & 0.7 & 0.7 \\
\hline $\mathrm{CP}$ & 1 & 2 & 4 & 0 & 0.3 & 0.4 & 0.8 & 1.1 \\
\hline Octyl & 1 & 2 & 4 & 3 & 0.9 & 1.1 & 1.6 & 3 \\
\hline C18 & 0 & 1 & 2 & 1 & 0.3 & 0.5 & 1.5 & 3.9 \\
\hline
\end{tabular}

Abbreviations: P3HT, poly(3-hexylthiophene); PF, polyfluorene.

${ }^{a}$ Calculated from the absolute value of the pristine sample (37\%).

${ }^{b}$ Calculated from the absolute value of the pristine sample $(0.3 \%)$

chains aggregation and maintained the homogeneous state. The use of fillers is a conventional strategy for tuning the properties of polymeric materials. It was found that the POSS fillers were also applicable for conjugated polymers. POSS are promising as efficient fillers for the production of robust opto-electronic materials for printed electronic devices. Furthermore, our findings mean that the organic-inorganic hybrids can be prepared not only by sol-gel methods but simply by the mixing of the polymers and POSS in common organic solvents followed by casting.

\section{CONFLICT OF INTEREST}

The authors declare no conflict of interest.

\section{ACKNOWLEDGEMENTS}

This work was partially supported by the Ube Industries Research Foundation (for KT) and a Grant-in-Aid for Scientific Research on Innovative Areas 'New Polymeric Materials Based on Element-Blocks (No. 2401)' (JSPS KAKENHI Grant Number JP25102521).

1 Teichler, A., Shu, Z., Wild, A., Bader, C., Nowotny, J., Kirchner, G., Harkema, S., Perelaer, J. \& Schubert, U. S. Inkjet printing of chemically tailored light-emitting polymers. Eur. Polym. J. 49, 2186-2195 (2013).

2 Tanaka, K. \& Chujo, Y. Advanced luminescent materials based on organoboron polymers. Macromol Rapid Commun. 33, 1235-1255 (2012).

3 Yoshii, R., Yamane, H., Nagai, A., Tanaka, K., Taka, H., Kita, H. \& Chujo, Y. $\pi$-Conjugated polymers composed of BODIPY or aza-BODIPY derivatives exhibiting high electron mobility and low threshold voltage in electron-only devices. Macromolecules 47, 2316-2323 (2014).

4 Kajiwara, Y., Tanaka, K. \& Chujo, Y. Enhancement of dye dispersibility in silica hybrids through local heating induced by the imidazolium group under microwave irradiation. Polym. J. 46, 195-199 (2014).

5 Okada, H., Tanaka, K., Ohashi, W. \& Chujo, Y. Photo-triggered molecular release based on auto-degradable polymer-containing organic-inorganic hybrids. Bioorg Med. Chem. 22, 3435-3440 (2014).

6 Kajiwara, Y., Nagai, A. \& Chujo, Y. Microwave-assisted synthesis of poly(2-hydroxyethylmethacrylate) (HEMA)/silica hybrid using in situ polymerization method. Polym. J. 41, 1080-1084 (2009).

7 Kajiwara, Y., Nagai, A. \& Chujo, Y. Microwave-assisted preparation of intense luminescent BODIPY-containing hybrids with high photostability and solvent resistance. J. Mater. Chem. 20, 2985-2992 (2010).

8 Kajiwara, Y., Nagai, A. \& Chujo, Y. Microwave-assisted one-pot synthesis of luminescent organic-inorganic hybrid via simultaneous process of sol-gel and Suzuki coupling reaction. Chem. Lett. 39, 480-481 (2010).

9 Kajiwara, Y., Nagai, A. \& Chujo, Y. Red/near-infrared emissive organic-inorganic hybrids doped with covalently bound boron dipyrromethene (BODIPY) dyes via microwave-assisted one-pot process. Bull. Chem. Soc. Jpn. 84, 471-481 (2011).

10 Kajiwara, Y., Nagai, A., Tanaka, K. \& Chujo, Y. Efficient simultaneous emission from RGB-emitting organoboron dyes incorporated into organic-inorganic hybrids and preparation of white light-emitting materials. J. Mater. Chem. C 1 , 4437-4444 (2013).
11 Okada, H., Tanaka, K. \& Chujo, Y. Preparation of environmentally resistant conductive silica-based polymer hybrids containing tetrathiafulvalen-tetracyanoquinodimethane charge-transfer complexes. Polym. J. 46, 800-805 (2014).

12 Okada, H., Tanaka, K. \& Chujo, Y. Regulation of responsiveness of phosphorescence toward dissolved oxygen concentration by modulating polymer contents in organic - inorganic hybrid materials. Bioorg. Med. Chem. 22, 3141-3145 (2014).

13 Tanaka, K. \& Chujo, Y. Chemicals-inspired biomaterials; developing biomaterials inspired by material science based on POSS. Bull. Chem. Soc. Jpn. 86, 1231-1239 (2013)

14 Tanaka, K. \& Chujo, Y. Advanced functional materials based on polyhedral oligomeric silsesquioxane (POSS). J. Mater. Chem. 22, 1733-1746 (2012).

15 Pielichowski, K., Njuguna, J., Janowski, B. \& Pielichowski, J. Polyhedral oligomeric silsesquioxanes (POSS) containing nanohybrid polymers. Adv. Polym. Sci. 201, 225-296 (2006).

16 Lickiss, P. D. \& Rataboul, F. Chapter 1 - Fully condensed polyhedral oligosilsesquioxanes (POSS): from synthesis to application. Adv. Organomet. Chem 57, 1-116 (2008).

17 Joshi, M. \& Butola, B. S. Polymeric nanocomposites-polyhedral oligomeric silsesquioxanes (POSS) as hybrid nanofiller. J. Macromol. Sci. Polym. Rev. 44, 389-410 (2004).

18 Zhang, W. \& Müller, A. H. E. Architecture, self-assembly and properties of well-defined hybrid polymers based on polyhedral oligomeric silsequioxane (POSS). Prog. Polym. Sci. 38, 1121-1162 (2013).

19 Ghanbari, H., Cousins, B. G. \& Seifalian, A. M. A nanocage for nanomedicine: polyhedral oligomeric silsesquioxane (POSS). Macromol. Rapid Commun. 32, 1032-1046 (2011).

20 Joshi, M. \& Butola, B. S. Isothermal crystallization of HDPE/octamethyl polyhedral oligomeric silsesquioxane nanocomposites: Role of POSS as a nanofiller. J. Appl. Polym. Sci. 105, 978-985 (2007).

21 Furgal, J. C., Jung, J. H., Goodson, T. III \& Laine, R. M. Analyzing structurephotophysical property relationships for isolated $T_{8}, T_{10}$, and $T_{12}$ stilbenevinylsilsesquioxanes. J. Am. Chem. Soc. 135, 12259-12269 (2013).

22 Furgal, J. C., Jung, J. H., Clark, S., Goodson, T. III \& Laine, R. M. Beads on a chain (BoC) phenylsilsesquioxane (SQ) polymers via $\mathrm{F}^{-}$catalyzed rearrangements and $\mathrm{ADMET}$ or reverse Heck cross-coupling reactions: Through chain, extended conjugation in 3-D with potential for dendronization. Macromolecules 46, 7591-7604 (2013).

23 Cordes, D. B., Lickiss, P. D. \& Rataboul, F. Recent developments in the chemistry of cubic polyhedral oligosilsesquioxanes. Chem. Rev. 110, 2081-2173 (2010).

24 Maegawa, T., Miyashita, O., Irie, Y., Imoto, H. \& Naka, K. Synthesis of a bi-functional terminal polyhedral octasilicate-core dendrimer containing carbazole and 1,8-naphthalimide, and its photoluminescence properties, film formability, and glass transition behavior. RSC Adv. 6, 8346-8353 (2016).

25 Kinoshita, S., Watase, S., Matsukawa, K. \& Kaneko, Y. Selective Synthesis of cis-trans-cis cyclic tetrasiloxanes and the formation of their two-dimensional layered aggregates. J. Am. Chem. Soc. 137, 5061-5065 (2015).

26 Sato, N., Kuroda, Y., Abe, T., Wada, H., Shimojima, A. \& Kuroda, K. Regular assembly of cage siloxanes by hydrogen bonding of dimethylsilanol groups. Chem. Commun. 51, 11034-11037 (2015).

27 Wang, L., Ishida, Y., Maeda, R., Tokita, M., Horiuchi, S. \& Hayakawa, T. Alkylated cage silsesquioxane forming a long-range straight ordered hierarchical lamellar nanostructure. Langmuir 30, 9797-9803 (2014).

28 Chiou, C.-W., Lin, Y.-C., Wang, L., Hirano, C., Suzuki, Y., Hayakawa, T. \& Kuo, S.-W. Strong screening effect of polyhedral oligomeric silsesquioxanes (POSS) nanoparticles on hydrogen bonded polymer blends. Polymers 6, 926-948 (2014).

29 Oguri, N., Egawa, Y., Takeda, N. \& Unno, M. Janus-cube octasilsesquioxane: facile synthesis and structure elucidation. Angew. Chem. Int. Ed. 55, 9336-9339 (2016).

30 Ayandele, E., Sarkar, B. \& Alexandridis, P. Polyhedral oligomeric silsesquioxane (POSS)-containingpolymer nanocomposites. Nanomaterials 2, 445-475 (2012).

31 Chujo, Y. \& Tanaka, K. New polymeric materials based on element-blocks. Bull. Chem. Soc. Jpn 88, 633-643 (2015). 
32 Jeon, J.-H., Tanaka, K. \& Chujo, Y. Rational design of POSS fillers for simultaneous improvements of thermomechanical properties and lowering refractive indices of polymer films. J. Polym. Sci. Part A: Polym. Chem 51, 3583-3589 (2013).

33 Yasumoto, Y., Yamanaka, T., Sakurai, S., Imoto, H. \& Naka, K. Design of low-crystalline and low-density isobutyl-substituted caged silsesquioxane derivatives by star-shaped architectures linked with short aliphatic chains. Polym. J. 48, 281-287 (2016).

34 Tanaka, K., Ishiguro, F. \& Chujo, Y. POSS ionic liquid. J. Am. Chem. Soc. 132, 17649-17651 (2010).

35 Tanaka, K., Yamane, H., Mitamura, K., Watase, S., Matsukawa, K. \& Chujo, Y. Transformation of sulfur to organic - inorganic hybrids employed by POSS networks and their application for the modulation of refractive indices. J. Polym. Sci. Part A: Polym. Chem. 52, 2588-2595 (2014).

36 Jeon, J.-H., Tanaka, K. \& Chujo, Y. Synthesis of sulfonic acid-containing POSS and its filler effects for enhancing thermal stabilities and lowering melting temperatures of ionic liquids. J. Mater. Chem. A 2, 624-630 (2014).

37 Jeon, J.-H., Tanaka, K. \& Chujo, Y. POSS Fillers for modulating thermal properties of ionic liquids. RSC Adv. 3, 2422-2427 (2013).

38 Tanaka, K., Jeon, J.-H., Inafuku, K. \& Chujo, Y. Enhancements of optical properties of dyes for bioprobes by freezing effect of molecular motion using POSS-core dendrimers. Bioorg. Med. Chem. 20, 915-919 (2012).

39 Tanaka, K., Ishiguro, F. \& Chujo, Y. Thermodynamic study of poss-based ionic liquids with various numbers of ion pairs. Polym. J. 43, 708-713 (2011).

40 Kubo, T., Koge, S., Ohshita, J. \& Kaneko, Y. Preparation of imidazolium salt type ionic liquids containing cyclic siloxane frameworks. Chem. Lett. 44, 1362-1364 (2015).

41 Tanaka, K., Adachi, S. \& Chujo, Y. Structure-property relationship of octa-substituted POSS in thermal and mechanical reinforcements of conventional polymers. J. Polym. Sci. Part A: Polym. Chem. 47, 5690-5697 (2009).

42 Yeo, H., Tanaka, K. \& Chujo, Y. Isolation of $\pi$-conjugated system through polyfluorene from electronic coupling with side-chain substituents by cardo structures. J. Polym. Sci. Part A: Polym. Chem. 50, 4433-4442 (2012).

43 Kawasaki, S., Yamada, M., Kobori, K., Jin, F., Kondo, Y., Hayashi, H., Suzuki, Y. \& Takata, T. Synthesis and chemical, physical, and optical properties of 9,9-diarylfluorene-based poly(ether-ether-ketone). Macromolecules 40, 5284-5289 (2007).
44 Leclerc, M., Diaz, F. M. \& Wegner, G. Structural analysis of poly(3-alkylthiophene)s. Makromol. Chem. 190, 3105-3116 (1989).

45 Tanaka, K., Adachi, S. \& Chujo, Y. Side-chain effect of octa-substituted POSS fillers on refraction in polymer composites. J. Polym. Sci. Part A: Polym. Chem. 48, 5712-5717 (2010).

46 Kakuta, T., Tanaka, K. \& Chujo, Y. Synthesis of emissive water-soluble network polymers based on polyhedral oligomeric silsesquioxane and their application as an optical sensor for discriminating the particle size. J. Mater. Chem. C 3, 12539-12545 (2015).

47 Tanaka, K., Ishiguro, F., Jeon, J.-H., Hiraoka, T. \& Chujo, Y. POSS ionic liquid crystals. NPG Asia Mater 7, e174 (2015).

48 Jeon, J.-H., Kakuta, T., Tanaka, K. \& Chujo, Y. Facile design of organic-inorganic hybrid gels for molecular recognition of nucleoside triphosphates. Bioorg. Med. Chem. Lett. 25, 2050-2055 (2015).

49 Tanaka, K. \& Chujo, Y. Unique properties of amphiphilic POSS and their applications. Polym. J 45, 247-254 (2013).

50 Kashiwagi, T., Inaba, A., Brown, J. E., Hatada, K., Kitayama, T. \& Masuda, E. Effects of weak linkages on the thermal and oxidative degradation of poly(methyl methacrylates). Macromolecules 19, 2160-2168 (1986).

51 Azuma, H., Asada, K., Kobayashi, T. \& Naito, H. Fabrication of $\alpha$ - and $\beta$-phase poly(9,9dioctylfluorene) thin films. Thin Solid Films 509, 182-184 (2006).

52 Bright, D. W., Dias, F. B., Galbrecht, F., Scherf, U. \& Monkman, A. P. The influence of alkyl-chain length on beta-phase formation in polyfluorenes. Adv. Funct. Mater. 19, 67-73 (2009).

53 Brinkmann, M. Structure and morphology control in thin films of regioregular poly(3hexylthiophene). J. Polym. Sci. B: Polym. Phys 49, 1218-1233 (2011).

54 Dang, M. T., Hirsch, L., Wantz, G. \& Wuest, J. D. Controlling the morphology and performance of bulk heterojunctions in solar cells. Lessons learned from the benchmark poly(3-hexylthiophene):[6,6]-phenyl- $\mathrm{C}_{61}$-butyric acid methyl ester system. Chem. Rev. 113, 3734-3765 (2013)

55 Chang, Y.-C., Hsiao, C.-C., Jen, T.-H., Liao, J.-L., Su, A.-C. \& Chen, S.-A. Effects of $\beta$ phase on light emission from polythiophenes-doped polyfluorene. J. Chin. Chem. Soc. 57, 564-574 (2010).

Supplementary Information accompanies the paper on Polymer Journal website (http://www.nature.com/pj) 